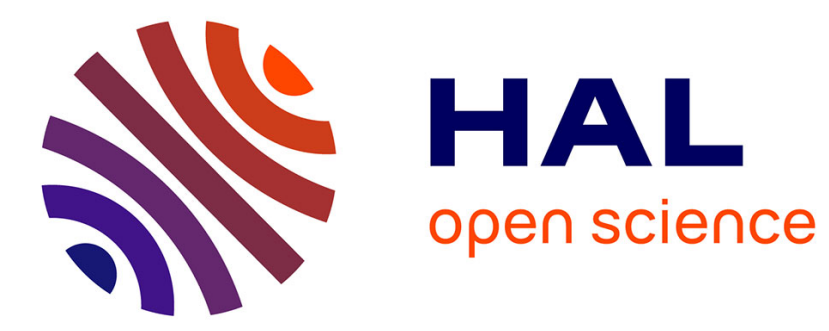

\title{
On the limits of DTN monitoring
}

Victor Ramiro, Emmanuel Lochin, Patrick Sénac, Thierry Rakotoarivelo

\section{To cite this version:}

Victor Ramiro, Emmanuel Lochin, Patrick Sénac, Thierry Rakotoarivelo. On the limits of DTN monitoring. IEEE 14th International Symposium on "A World of Wireless, Mobile and Multimedia Networks" (WoWMoM), Jun 2013, Madrid, Spain. pp.1-6, 10.1109/WoWMoM.2013.6583441 . hal02552554

\section{HAL Id: hal-02552554 \\ https://hal.science/hal-02552554}

Submitted on 23 Apr 2020

HAL is a multi-disciplinary open access archive for the deposit and dissemination of scientific research documents, whether they are published or not. The documents may come from teaching and research institutions in France or abroad, or from public or private research centers.
L'archive ouverte pluridisciplinaire HAL, est destinée au dépôt et à la diffusion de documents scientifiques de niveau recherche, publiés ou non, émanant des établissements d'enseignement et de recherche français ou étrangers, des laboratoires publics ou privés. 


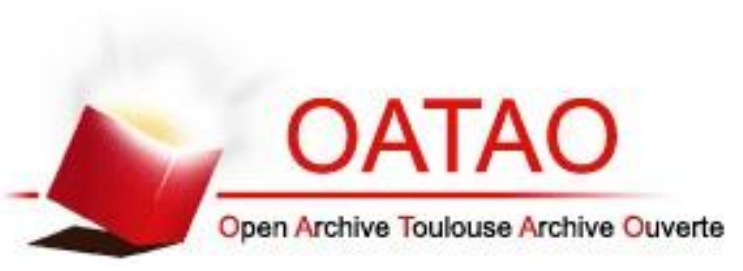

\section{Open Archive Toulouse Archive Ouverte (OATAO)}

OATAO is an open access repository that collects the work of Toulouse researchers and makes it freely available over the web where possible.

This is an author-deposited version published in: http://oatao.univ-toulouse.fr/ Eprints ID: 9122

To cite this document: Ramiro, Victor and Lochin, Emmanuel and Sénac, Patrick and Rakotoarivelo, Thierry On the limits of DTN monitoring. (2013) In: IEEE AOC'13 - The Seventh IEEE WoWMoM Workshop on Autonomic and Opportunistic Communications, 04-07 Jun 2013, Madrid, Spain.

Any correspondence concerning this service should be sent to the repository administrator: staff-oatao@inp-toulouse.fr 


\title{
On the limits of DTN monitoring
}

\author{
Victor Ramiro $^{\dagger}$, Emmanuel Lochin ${ }^{\dagger}$, Patrick Senac ${ }^{\dagger}$, Thierry Rakotoarivelo ${ }^{\ddagger}$ \\ ${ }^{\dagger}$ ISAE/University of Toulouse, Toulouse, France \\ ${ }^{\ddagger}$ National ICT Australia (NICTA), Alexandria, Australia
}

\begin{abstract}
Compared to wired networks, Delay/Disruption Tolerant Networks (DTN) are challenging to monitor due to their lack of infrastructure and the absence of end-to-end paths. This work studies the feasibility, limits and convergence of monitoring such DTNs. More specifically, we focus on the efficient monitoring of intercontact time distribution (ICT) between DTN participants. Our contribution is two-fold. First we propose two schemes to sample data using monitors deployed within the DTN. In particular, we sample and estimate the ICT distribution. Second, we evaluate this scheme over both simulated DTN networks and real DTN traces. Our initial results show that $(i)$ there is a high correlation between the quality of sampling and the sampled mobility type, and (ii) the number and placement of monitors impact the estimation of the ICT distribution of the whole DTN.
\end{abstract}

Keywords-DTN, Monitoring, Temporal random walk, Measurement.

\section{INTRODUCTION}

Monitoring is an important task in networking. Indeed, knowing the state of the network allows one to apply decisions for optimizing and adapting communication to the context. Monitoring over a DTN network should allow a fair tradeoff between the complexity and the scalability of the network characterization. The natural temporal sampling of monitoring provides a method to reduce the significant amount of data that is needed to characterize some network feature or parameter. This may be useful to improve DTN algorithms.

DTN monitoring and particularly the deployment of a monitoring infrastructure raises numerous challenging issues. For instance the notion of end-to-end path cannot be applied. Moreover, opportunistic communication coupled with potential network partitioning makes very challenging the construction by some nodes of a consistent view of the network "state". DTN monitoring raises also the issue of the choice of the metrics to monitor in order to make it possible for each node to get as lightly and as quickly as possible a consistent and useful view of the network dynamics. In such dynamic context several questions arise. In particular, how many and which nodes to select to perform an efficient monitoring? Furthermore, given the mobility of nodes, can we define where to monitor and how the monitors should move conjointly with other nodes? In summary monitoring a DTN network combines issues about temporal, spatial and nodes sampling that make difficult finding an efficient solution. Finally, monitoring can help to understand how the network evolves and when phase changes occur, providing a bigger resilience to changes.

The intercontact time distribution (ICT) is a global characterization of the DTNs [1] that delivers an abstraction of the network complexity (e.g. mobility model, number of nodes). Routing protocols, such the Spray and Wait [2] protocol, can leverage on this parameter to adapt their behavior for insuring some performances. The problem of evaluating intercontact time just by monitoring this parameter from some networks nodes is not trivial. Today, the common way to perform such evaluation is to drive a statistical analysis of collected intercontact time data during a certain period of time between all the nodes [1], [3]. Usually, this process is cumbersome and not scalable when the increasing number of nodes. Furthermore, the resulting statistical analysis gives an averaged view of the network during a given period.

In this work we study the feasibility and limits of DTN monitoring. Our main contributions are the following:

1) DTN Monitoring: we introduce the DTN monitoring problem. We explain why the common network monitoring approach does not completely suit in the DTN case. We provide some insights on the impact of time dependence in the metrics we can monitor. We propose two methods to study the impact of the DTN connection dynamics for monitoring: static monitoring and dynamic monitoring.

2) Limits and convergence of ICT monitoring: we study the ICT monitoring and how it can be presented as a sampling problem. We provide both simulations and real human traces analysis to study the convergence of the proposed sampling methods. We study those limits in terms of the total cost of deployment (number of nodes monitored, memory cost)

Our main contribution is to weight up the trade-off between the complete statistical analysis and the approximation given by the monitoring model for the ICT.

The paper is structured as follows: in section II we drive an analysis on the feasibility of monitoring a DTN network. In section III we present our ICT sampling method. In sections IV and $\mathrm{V}$ we test our algorithms over simulated and real DTNs. We determine how many nodes are needed to perform the monitoring (section VI). Later in section VII we present some related work and we conclude in VIII.

\section{DTN MONITORING}

This section presents the problem statement for DTN monitoring and provides some key insights to characterize possible solutions.

\section{A. Why monitoring?}

Since there are several opportunistic routing algorithms, deploying a DTN assumes an agreement between networks 
nodes on the routing optimization strategy and resulting selected protocols. A newcomer in the network needs some hints about the network features and dynamics for applying wise routing decisions. Previous studies [1] showed that routing protocol selection and configuration can be done knowing the intercontact law between them. Having this characterization allows to select both the right algorithm and the right configuration parameters to diffuse the information on the network. Nevertheless, considering that this information is dynamic this type of information has to be continuously monitored and updated among the nodes.

\section{B. What is DTN monitoring?}

DTN monitoring raises different challenges than infrastructured wired/wireless network. This is due to the fact that: (i) in a DTN there is no notion of end to end path defined as a sequence of contiguous and contemporary links, (ii) nodes and links spatio-temporal evolution plunges monitoring in a highly dynamic context and (iii) introducing new nodes or modifying some nodes behavior for monitoring purposes potentially changes contact patterns and the observed and monitored parameter.

\section{How to monitor?}

Monitoring a DTN is a complex process. We have already stated the necessity to be non obtrusive to monitor contacts in a DTN. This raises the question of monitoring nodes' placement and movement. We can "attach" our monitors to a subset of nodes and follow them. Hence we will capture any contact the node has with other peers. This attachment can be formally expressed by a time mapping function map that defines at any time the set of monitoring nodes.

For instance, Figure 1 depicts the mapping function map = $\left\{\left(M_{1}, 1\right),\left(M_{2}, 2\right),\left(M_{3}, 7\right)\right\}$. This mapping function entails two layers of interactions, respectively the nodes plane and the monitors plane. In the nodes plane we see all the existing spontaneous links between nodes, for instance the node 1 is connected with nodes 2 and 3 . Since node 1 is associated with monitor $M_{1}$ by the mapping function, the later in the monitoring plane will capture both established connections. We can see in red all the contacts that we are able to capture with the current mapping. Notice that this mapping can be a function of time in order to follow the evolution of the network dynamic. Hence in a posterior time we might see $M_{1}$ associated with another node than 1 . This leads us to introduce the following distinction:

- Static monitoring: when the association between nodes and monitors is invariant over the observation time;

- Dynamic monitoring: when there is a time depending process that associates nodes and monitors over the observation time.

The idea behind this distinction is that in the first case we keep a regular monitor strategy; while in the second we introduce the connections dynamics to provide diversity. Nevertheless, this diversity may have an associated cost. Given a metric and a time depending process, we may need to engage

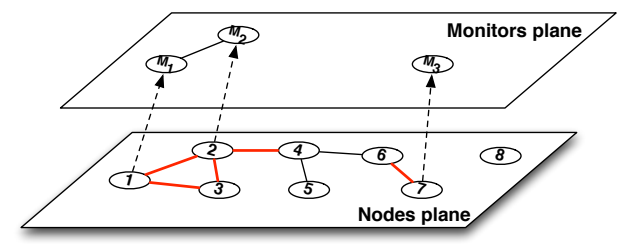

Figure 1: Monitor architecture in DTN

the nodes in the node plane to keep extra information in memory to perform the monitoring. Notice that the notion of static and dynamic monitoring is related with the monitoring process evolution and not with the movement of the nodes: a static monitor does not imply its associated node does not move in the space.

We can also leverage the fact that in the monitor plane we can also have spontaneous contacts between monitor. Since each monitor will potentially monitor disjoint areas of the DTN, we need to define a way to exchange information between monitor nodes contacts. Exchanging this information between monitors may be resource consuming, therefore we propose this exchange policy must be done taking into account two key factors: (i) time relevance (exchange fresh information if possible) (ii) summarized information (prefer summarized metrics if possible).

Finally, defining the mapping function is not trivial. In the following we will use these two strategies and assess their capacity to deliver an accurate estimation of the $I C T$ distribution. We study their complexity and resource use.

\section{Which metric to monitor?}

Several parameters can potentially offer a monitoring interest in a DTN in order to get an as complete as possible view of the network state. One can cite, the number of active nodes, the diameter of the network, the delivery ratio. We already underlined the importance of the knowledge of ICT distribution for applying wise routing decisions. Therefore in the rest of this paper we will focus on ICT monitoring. However the promoted monitoring approaches are generic enough to be used for monitoring a wide range of parameters.

\section{A SIMPLE MONITORING MODEL FOR ICT}

This section presents the model basics to characterize the intercontact time. We define some basic notation and then we explain how to build the approximate characterization of the intercontact time. Then we define what we understand as intercontact time approximation.

\section{A. Model basics}

Given the set $\mathcal{N}=\{1,2, \ldots, n\}$ of nodes and a discretized time set $\mathcal{T}=\left\{t_{1}, t_{2}, \ldots, t_{T}\right\}$, we model a DTN as a finite set of graphs snapshots $\mathcal{G}=\left\{G_{t_{1}}, G_{t_{2}}, \ldots, G_{t_{T}}\right\}$, where $G_{t_{i}}$ represents the connection graph at time $t_{i}$. The time window $\delta=t_{i+1}-t_{i}, \forall i$ defines the granularity with which we observe at a discrete level the evolution of the network. 

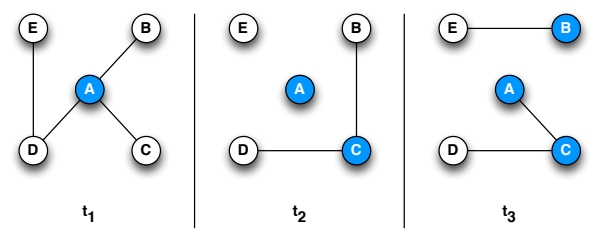

Figure 2: Evolution of the dynamic random walk process

We define the intercontact time for two distinct nodes $(i, j)$ as the time elapsed between two successive contacts between them. The pairwise intercontact time distribution $I C T_{(i, j)}(t)$ is the intercontact times we observe for a pair $(i, j)$ along the time $T$. The global intercontact time $I C T(t)$ is the $e c d f^{1}$ for $\cup_{i, j} I C T_{(i, j)}(t)$. The set of connections defined by $(i, j)$ may not necessarily be the same defined by $(j, i)$. This is due to the inherent behavior of some wireless protocols or because measuring problems. In this work we assume that the pairwise distribution for $(i, j)$ and $(j, i)$ are the same. By definition we see that to characterize the global $I C T(t)$ we need to know all the pairwise $I C T_{(i, j)}(t)$ distributions. Due to the difficulty to gather all the pairwise distributions, we propose to select a subset of nodes that can approximate the global $I C T$ distribution ${ }^{2}$.

Finally, the mapping function is defined as map $: \mathcal{M} \times \mathcal{T} \rightarrow$ $\mathcal{N}$, where $\mathcal{M}$ is the set of available monitors.

\section{B. ICT monitoring: what do you mean?}

Since $I C T$ is defined as a probability distribution, the problem is reduced to construct an estimation of this distribution. We define as $\langle I C T\rangle$ the estimation of the $I C T$ distribution delivered by the monitoring process. In the following, we propose to investigate methods providing representative $\langle I C T\rangle$ sampling of the whole network. Notice that we can generalize this method to $|\mathcal{M}|$ monitors. Each one will gather $\langle I C T\rangle_{k}$. It follows that $\langle I C T\rangle=\cup_{k}\langle I C T\rangle_{k}$.

1) Static monitoring: We define $\mathcal{S}$ as a random sample of size $|\mathcal{M}|$ from the set $\mathcal{N}$. The mapping is defined associating each node from the random sample to an available monitor for the whole duration of the monitoring process. Formally map : $M_{i} \rightarrow i \quad \forall i \in \mathcal{S}$. Notice that this relationship is not time dependent, hence we say the monitor nodes are static.

2) Dynamic monitoring: In this case we use a temporal random walk to define the mapping function map for each step of the monitoring process (III-C, III-D). In the case of multiple monitors we assume that we do not map more than one monitor per node to maximize the sampling coverage of the monitoring. Of course in this approach we do not know where will finish each token. We do not deal with the tokens' recollection problem in this paper.

\footnotetext{
${ }^{1}$ ECDF: empirical cumulative distribution function

${ }^{2}$ Notice the abuse of notation for the $I C T$ dataset and its associated $I C T(t)$ function. We will treat them both indistinguishable.
}

\section{Temporal random walks}

Random walks are a simple and well known way to sample static graphs [4], [5], [6]. Some extensions propose to either run in parallel with multiple walkers [5] or to study the temporal case [6]. Our proposed monitoring scheme is based on a multi-temporal random walk which combines these two approaches.

A random walk in a static graph starts from one node of the network. Then the walk progresses choosing with uniform probability among the edges that are connected. The process of selecting randomly the edges will deliver both: a sample of edges and a subset of nodes (the ending of the selected edges).

In the dynamic graphs or networks case, we define a random walk process through the temporal graph. We select a starting node $n_{1}$. After $\gamma$ time we randomly select one of its current neighbors and walk towards this node. We repeat this process at rate $\gamma$ starting from the last selected node. Therefore a temporal random walk is able to walk along dynamic path that does not exist in any timed graph of the dynamic graph. In the following we will consider that $\gamma=\delta$.

For instance, in Fig. 2 we see that at time $t_{1}$ we select $A$ as starting node. At this time, the $A$ node is connected with nodes $\{B, C, D\}$. Randomly $C$ is selected. Then at time $t_{2}$ the connections have changed. We can see that $C$ is connected with nodes $\{B, D\}$. In this case we select randomly the node $B$. Finally at time $t_{3}$ we can just jump into the node $E$. Here we notice that in the temporal random walk we can profit from temporal paths that are created with the evolution of the communication: the path between $\mathrm{A}$ and $\mathrm{E}$ just exists in terms of other nodes contacts.

\section{Temporal random walks to sample ICT}

To understand the monitoring selection process we can take the image of a token that can be passed among the nodes as a right to monitor. While a node holds the token it will have associated a monitor and record the intercontacts in the token. Then the token will be passed by the temporal random walk and hence $\langle I C T\rangle$ will be collected. In Fig. 2 we see that the token is passed among nodes in the following sequence: $\left\{A \stackrel{t_{1}}{\longrightarrow} C, C \stackrel{t_{2}}{\longrightarrow} B, B \stackrel{t_{3}}{\longrightarrow} E\right\}$ and hence the mapping function will be map $=\left\{\left(\left(M_{1}, t_{1}\right), A\right),\left(\left(M_{1}, t_{2}\right), C\right),\left(\left(M_{1}, t_{3}\right), B\right)\right\}$. Here $\langle I C T\rangle$ is constructed as the union of the intercontact time values sampled by the token at each connection $(i, j)$.

Since the rate of the temporal random walk is the same than the granularity of the network evolution $(\gamma=\delta)$, the probability to sample an intercontact time among two nodes is 0 (the token will walk out from the node before the two nodes are in contact again). Instead we propose the following extension: in the node plane we keep a memory of the contacts we had in the past and we use the temporal random walk to recollect the sampling data from that memory. We propose three strategies to compare the impact of the associated memory and selection process. Given a node $i$ holding the token at time $t$ the sampling is defined as:

- Last intercontact time: where we just consider the last valid intercontact time between $(i, j) \forall j$ connected with 
$i$ at the current time $t$. This implies that each node must keep a memory of the last contact with any node.

- All intercontact time: where we add the history of past intercontact times that has not been already considered between $(i, j) \forall j$ connected with $i$ at current time $t$. In this case the memory is extended to all the non recorded contacts in the token, which is in the worst case all the period of observation.

- Any intercontact time: where we add the history of past intercontact times that has not been already considered with any other node $k$. In this case we keep the same memory than for the All method, but we add any non recorded information in the token, even if there is not a connection between $(i, k)$ at the current time $t$.

\section{E. ICT approximation: how to compare?}

We use the two-sample Kolmogorov-Smirnov (KS) statistical test to compare the sampled $\langle I C T\rangle$ from the original ICT. The KS test defines a distance $D$ (Eq. 1) between these distributions to determine if they are drawn from a similar underlying distribution (i.e. the null hypothesis).

$$
D=\sup _{t}|I C T(t)-\langle I C T\rangle(t)|
$$

We use the p-value to reject the null hypothesis with significance level $\alpha=5 \%$. Rejecting the null hypothesis means that both samples definitely do not come from the same distribution. Failing to reject means that both distributions are the same within an error associated to the significance level.

\section{Simulation EXPERIMENTS}

In this section we perform a series of simulations with "The ONE Simulator" [7] to test the intercontact time monitoring strategies presented in Section III.

\section{A. RWP simulation setup}

For each simulation we setup a group of 100 nodes moving according to the random waypoint movement (RWP) in a square of $100 \times 100 \mathrm{~m}^{2}$. We gather the approximated intercontact time distribution according to the four methods presented in Section III-B: $\langle I C T\rangle_{\text {static }},\langle I C T\rangle_{\text {last }},\langle I C T\rangle_{\text {all }}$, $\langle I C T\rangle_{\text {any }}$. For the simulations we test with $N=100$ nodes and $\delta=5$ minutes for $T=500$ time windows. Since we want to study the limit of the monitoring methods, for each one we increase the number of monitors/tokens from 1 to 100 . We repeat each simulation 10 times to reduce the randomness effects.

\section{B. Simulations analysis}

We observe that in the RWP simulation each monitor has an indistinguishable view of its surrounding: the monitor see that all nodes move uniformly into space. In Fig. 3a, we graph the average distance (Eq. 1) as a function of the number of monitors. As expected, we observe that the more monitors we add, the smaller the distance we obtain (hence the better

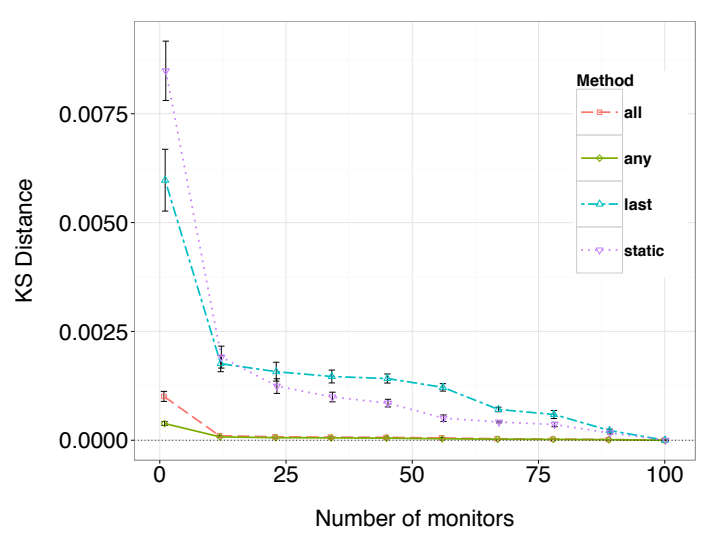

(a) KS distance for RWP traces

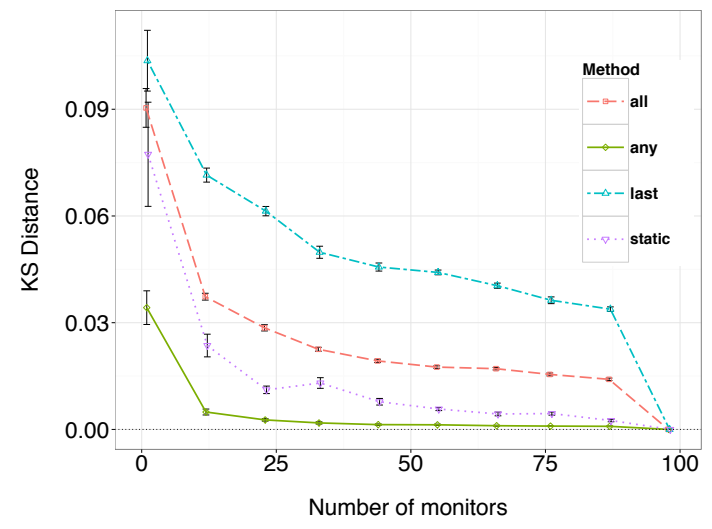

(b) KS distance for INFOCOM traces

Figure 3: KS Distance with increasing number of monitors (RWP simulation and INFOCOM traces). Notice that in both cases, the lower bound is obtained using dynamic monitoring with the any strategy (full memory and interchanges)

the approximation). This is independent of the method used. Since the homogeneous view in the RWP model, we can say that increasing the number of monitors increases the contacts and hence the quality of the approximation. Also, we see that in the case of using just one monitor, it is better to use the dynamic mode than the static one. This is due the fact that we gather more contacts when we move. With the same argument we see that the distance between the different strategies in the dynamic mode is correlated with respect the amount of information we add in the sample: $D\left(\langle I C T\rangle_{\text {any }}\right)<$ $D\left(\langle I C T\rangle_{\text {all }}\right)<D\left(\langle I C T\rangle_{\text {last }}\right)$. When calculating the $\mathrm{KS}$ test, we always verify the null hypothesis for any number of monitors. This is independently of the selected method (static and dynamic) and sampling strategy selected (last, all, any). In the case of RWP we know that the ICT [8] follows an exponential law, we can fit an exponential model to $\langle I C T\rangle$ and obtain the desired result. As overall conclusion: in the random waypoint scenario, we can monitor a group of nodes using a subset of monitors. The key parameter to take into account is the number of contacts, that can be regulated either 

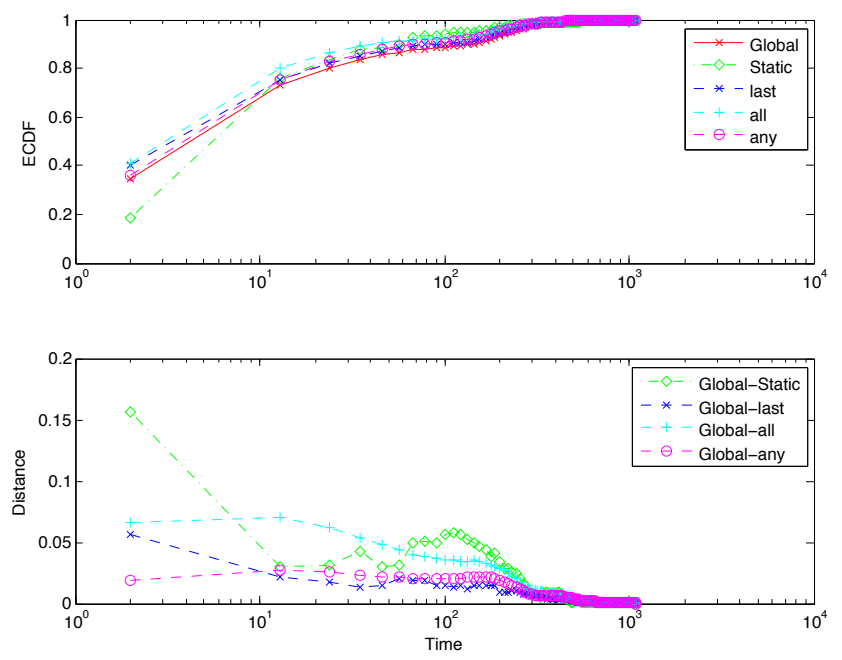

Figure 4: Up: Monitoring algorithms comparison for the INFOCOM ICT sampling case. Down: Distance between $I C T$ and $\langle I C T\rangle$. (Notice the log scale on the $\mathrm{x}$-axis)

by increasing the time sampling or by increasing the number of nodes in the space.

\section{REAL TRACES EXPERIMENTS}

In this section, we analyze the algorithms presented in section III-B with real traces.

\section{A. Traces setup}

We use the INFOCOM traces [9] to study the 98 distributed nodes in the conference. We aggregate the traces into snapshots with $\delta=5$ minutes. We also impose the symmetry of the connections.

\section{B. Traces Analysis}

We perform the same analysis than in IV-B. As expected, in Fig. 3b we see that with both methods and with any sampling strategy, we always decrease the distance while adding monitors. However, we see that the dynamic method with the last and all sampling strategies are lower bounded by the static method. This is equivalent to say that randomly selecting a number of monitors and staying statically attached to them provides a better approximation than dynamically changing nodes. In the dynamic case we observe we get a bigger distance due the fact that passing the token at each time can bias the data to smaller values of intercontact time (we will have a higher probability of short intercontact times than the real $I C T$ as show in Fig. 4). In the static case we have a partial local view of the network, but consistent within all the period of observation. This will add longer intercontact times to the sampling reducing the bias (we will add more information to the tail of the distribution reducing the probability of shorter

\begin{tabular}{|c|c|c|c|c|}
\hline & Static & Last & All & Any \\
\hline Average case & $>17 \%$ & $>98 \%$ & $>95 \%$ & $>3 \%$ \\
\hline Worst case & $>78 \%$ & $>98 \%$ & $>95 \%$ & $>15 \%$ \\
\hline \hline Memory & $O(1)$ & $O(N)$ & $O(N T)$ & $O(N T)$ \\
\hline
\end{tabular}

Table I: Monitors coverage (in percentage of nodes in the DTN) needed to be sure that $\langle I C T\rangle$ is a good estimator for $I C T$ (i.e. stop rejecting the null hypothesis)

intercontact times). In other words, adding diversity is not enough to improve the sampling because it add bias.

Finally, we can see that in all cases, the dynamic method with the any sampling strategy draws the smaller approximation distance. This is due the fact that this method is a mix between static and dynamic monitoring. Indeed, the holder of the token at the last snapshot will add all its intercontact time information. This information is equivalent to the information that he would have added as a static monitor. However, we have to remember that the cost of this strategy requires that all the nodes in the plane node store theirs contacts. Here the token becomes just a method of data recollection. An obvious improvement is to leverage the monitors connections. When they receive the token they may add their information as well as their past connections information.

\section{DISCUSSION}

Both, simulations and trace analysis confirm the possibility to select a group of nodes and attach them monitors to characterize the global behavior. These experiments lead us to conclude that it is not possible to define the most representative set of monitors: any non random selection will introduce bias to the sampling (Fig. 4). Nevertheless we have not yet explained the limits of the monitoring. In Table I, we show the increasing percentage of nodes needed to stop rejecting the null hypothesis $(p v a l>\alpha)$. Stop rejecting the null hypothesis implies that $\langle I C T\rangle$ is a good estimator for the real $I C T$. Since we repeated the experience 10 times, we provide two cases: ( $i$ ) average case (average pval) (ii) worst case (minimum pval). Notice that the worst case is more strict in the sense that we had pval $>\alpha$ for all the repetitions. We also add the memory cost for each node: (i) static: keeping no extra information, (ii) last: keeping the list of the last intercontacts and (ii) all/any: keeping the whole past history of intercontacts. We see that in the static case we need to cover at least $75 \%$. This implies a huge cost for monitoring. On the other hand in the dynamic case we need to cover $15 \%$ when we use the any sampling strategy. Nevertheless this number hides the fact that all nodes in the nodes plane must be storing in memory the past connections. We thus obtain the trade-off sought: either we add more memoryless monitors or we have less monitors to recollect the data of nodes with higher memory capacity. If we accept a non statistically accurate view this numbers drop to $17 \%$ and $3 \%$ respectively.

Using this reasoning for the INFOCOM conference, and assuming that the devices delivery was random, we conclude that to get a statistically representative estimation of the $I C T$, 
the experience should have covered at least $75 \%$ of the people in the conference.

Finally we see that the best case is the dynamic mode with the any sampling strategy. This is indeed the limit of the monitoring: when the monitors plane and the nodes plane become just one, and all nodes are constantly monitoring and storing their neighborhood and we use the random walk process just to recollect data.

\section{RELATED WORK}

On sampling static graph structures, we have that sampling a scale free network is not scale free [10]. In this work they present a simple selection that does not respect the inner structure of the network. Sampling from large graphs [4] obtain that more than the half of the nodes are needed to obtain a good sample. This support our result than just performing random sampling or basic variations of random walk is not enough and the cost of the monitoring is high. Multiple random walk [5] introduces the idea of variability in the starting point in order to avoid some known bias of random walks. Finally [6] present a complete characterization of random walk in temporal networks. This work reviews several metrics that can be obtained from temporal random walks. However there is no comparison in terms of quality of the sampling w.r.t the original temporal graph.

On the DTN monitoring side, in [11] there is an extension off well known aggregation algorithms for connected networks. Specifically the notion of pairwise averaging and populations protocols to the DTN scenario. However this work does not provides a mean to measure the error of the estimation. Instead the estimation is just performed by a given amount of time or by a given number of contacts desired, assuming that the more contacts you have, the better the estimation will be. Most of the works that characterize a DTN are based in the global estimation of intercontact time. In [12] present an analytical model which derives from pairwise intercontact time distribution the aggregated distribution of the group. Remarkably, this study shows that there is no exact mirror between the pairwise connection with the aggregated distribution. If we assume that pairwise distributions follows an exponential law, then the aggregated distribution will follow a power law. Finally [13] present a vicinity study to characterize the behavior of the DTN. In this work is present the concept of $k$-vicinity as all the nodes that are at most at $\mathrm{k}$ hops from a given node and the $k$ intercontact which is the time while a node left and come back to a given $k$-vicinity. Trace analysis shows that $\mathrm{k}$-vicinities intercontact time follows power laws with exponential decay after a given time. Moreover, the $k$-vicinity of size $\mathrm{k}=\{2,3\}$ gives already enough awareness to a node of its surrounding. This assumption is supported in the existence of groups in the node movements.

\section{CONCLUSION}

This paper presents our initial study on the feasibility and limits of monitoring DTN. We first defined monitoring in the DTN context, highlighted some of the challenges, and discussed potential metrics to monitor. We then presented our main contributions, namely a simple set of methods to monitor ICT and their evaluations using both simulated and real trace based DTNs. Our results show that it is possible to approximate the ICT characteristic of a DTN with only collecting a subset of that global information. However, this has a cost in terms of number of monitor or states of past activity to track. This tradeoff in monitor numbers and memory capabilities have an impact on the quality of the estimated ICTs. We further discussed this tradeoff and proposed some potential approach to take (e.g. favoring monitor number or memory capacity)

In the future we plan to develop a reflective monitoring system for DTNs. We showed evidence to support the idea that a monitor system is not accurate enough to provide the intercontact time approximation. We seek to study under which conditions certain networks can converge to a uniform state where all the nodes share a good approximation of the global network. This can be exploited to improve algorithms over a DTN from local information.

\section{ACKNOWLEDGMENTS}

The authors would like to thank Anh-Dung Nguyen for his ideas to improve this work. This work is partially supported by CONICYT Becas Chile PhD program and by the CUDEN STIC-AmSud project.

\section{REFERENCES}

[1] V. Conan, J. Leguay, and T. Friedman, "Characterizing pairwise intercontact patterns in delay tolerant networks," in Autonomics, 2007.

[2] T. Spyropoulos, K. Psounis, and C. S. Raghavendra, "Spray and wait: an efficient routing scheme for intermittently connected mobile networks," in $A C M W D T N, 2005$.

[3] A. Chaintreau, P. Hui, J. Crowcroft, C. Diot, R. Gass, and J. Scott, "Impact of human mobility on the design of opportunistic forwarding algorithms," in INFOCOM, april 2006.

[4] J. Leskovec and C. Faloutsos, "Sampling from large graphs," in $K D D$. New York, NY, USA: ACM, 2006.

[5] B. Ribeiro and D. Towsley, "Estimating and sampling graphs with multidimensional random walks," in $I M C$. Melbourne, Australia: ACM, 2010, pp. 390-403.

[6] M. Starnini, A. Baronchelli, A. Barrat, and R. Pastor-Satorras, "Random walks on temporal networks," Phys. Rev. E, vol. 85, p. 056115, May 2012.

[7] A. Keränen, J. Ott, and T. Kärkkäinen, "The ONE Simulator for DTN Protocol Evaluation," in SIMUTools '09. New York, NY, USA: ICST, 2009.

[8] R. Groenevelt, P. Nain, and G. Koole, "The message delay in mobile ad hoc networks," Perform. Eval., vol. 62, no. 1-4, pp. 210-228, Oct. 2005.

[9] J. Scott, R. Gass, J. Crowcroft, P. Hui, C. Diot, and A. Chaintreau, "CRAWDAD trace cambridge/haggle/imote/infocom," Jan. 2006.

[10] M. P. H. Stumpf, C. Wiuf, and R. M. May, "Subnets of scale-free networks are not scale-free: Sampling properties of networks," Proc. of the National Academy of Sciences of the USA, 2005.

[11] A. Guerrieri, I. Carreras, F. De Pellegrini, A. Montresor, and D. Miorandi, "Distributed estimation of global parameters in delay-tolerant networks," in IEEE WoWMoM, jun 2009.

[12] A. Passarella and M. Conti, "Characterising aggregate inter-contact times in heterogeneous opportunistic networks," in NETWORKING, 2011, vol. 6641, pp. 301-313.

[13] T. Phe-Neau, M. Dias de Amorim, and V. Conan, "Vicinity-based dtn characterization,” in ACM MobiOpp, March 2012. 